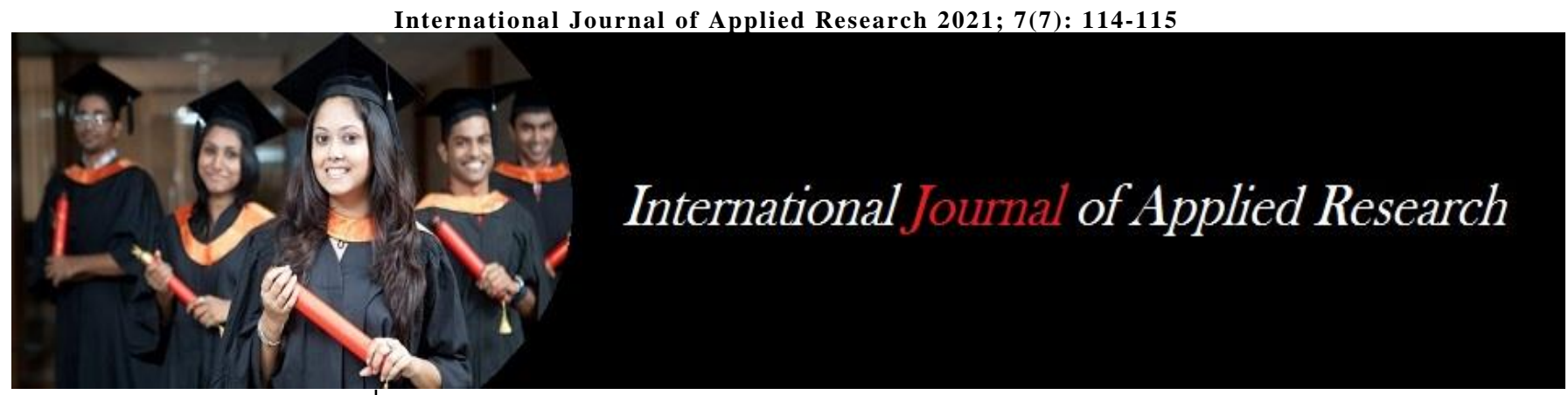

ISSN Print: 2394-7500

ISSN Online: 2394-5869

Impact Factor: 8.4

IJAR 2021; 7(7): 114-115

www.allresearchjournal.com

Received: 05-05-2021

Accepted: 04-06-202

Dr. Nirmalya Roy

Professor in the department of

Medicine KPCMCH, Kolkata,

West Bengal, India

Dr. Suman Sarkar

2nd Year Resident in the department of Medicine

KPCMCH, Kolkata,

West Bengal, India

Dr. Ankan Pathak

3rd year Resident in the

department of Medicine

KPCMCH, Kolkata,

West Bengal, India

Dr. Nikhil Sonthalia

lst year Resident in the department of Medicine

KPCMCH, Kolkata,

West Bengal, India
Corresponding Author: Dr. Suman Sarkar 2nd Year Resident in the department of Medicine KPCMCH, Kolkata, West Bengal, India

\section{A unique case report of successful pregnancy and delivery after being treated with pioglitazone and glimepiride (In first three months)}

\author{
Dr. Nirmalya Roy, Dr. Suman Sarkar, Dr. Ankan Pathak and Dr. Nikhil \\ Sonthalia
}

DOI: https://doi.org/10.22271/allresearch.2021.v7.i7b.8739

\begin{abstract}
A planned pregnancy (with IVF) in a patient with type 2 diabetes was treated with insulin along with glimepiride and pioglitazone (treated in first three months) from pre-conception stages until the postpartum is reported. She delivered a single viable male child at 33 weeks of gestation due to pregnancy induced hypertension and type 2 diabetes mellitus with no abnormalities. The newborn was healthy without any congenital and other abnormalities. To our belief, this is the first case reporting the use of pioglitazone as well as glimepiride in early pregnancy. It should be noted that both of the drugs are not recommended to be used in pregnancy but we may come across situations where the patient is already exposed to these drugs and not willing to abort pregnancy and this case adds value to the existing body of medical literature in such cases.
\end{abstract}

Keywords: Pioglitazone, abnormalities, dangerous, reporting, hypoglycemia, glimepiride, hypertension

\section{Introduction}

Pregnancy with Type 2 diabetes is a dangerous combination. If left untreated or undertreated, it can result in a number of complications which can affect both the mother and the child ${ }^{[1]}$. The complications can be pre-eclampsia, polyhydramnios, fetal macrosomia, increased risk of hypoglycemia along with other pre-natal, peri-natal and post-natal complications ${ }^{[2]}$. Treating diabetes in pregnancy is a difficult task and so the goal is to adequately control the glycemic values and thereby reduce the complications ${ }^{[3]}$. As we are left with limited options to treat diabetes in pregnancy, one can say for sure that insulin is the gold standard for the same due to lack of long term safety data for other agents. Acceptable alternatives now include some oral hypoglycemic agents like metformin and glyburide. Most of the drugs used in pregnancy crosses the placenta which can have a teratogenicity effect on the growing fetus but glyburide does not cross the placenta ${ }^{[4]}$. Metformin despite crossing placenta have good outcomes like less maternal weight gain and increased patient acceptability. Glyburide increases secretion of insulin and by lowering glucose toxicity reduces insulin resistance. Thiazolidinediones use in pregnancy is not recommended as there is paucity of data supporting it's use. We report a planned pregnancy in type 2 diabetes patient from pre-conception stages until post-partum who was treated with glimepiride and pioglitazone until 3rd month of pregnancy, both of which are not recommended by clinical practice guidelines.

\section{Case presentation}

A 48-year-old female patient of Type 2 diabetes Mellitus for past 14 years and hypertension for 7 years. She conceived via IVF (In vitro fertilization) and presented to us at the 3rd month of pregnancy with poor glycemic levels (FBS - $146 \mathrm{mg} / \mathrm{dL}, \mathrm{PP}-274 \mathrm{mg} / \mathrm{dL}$ ). She was on Glimepiride and Pioglitazone when she reported to us. The risks of conception were discussed with the patient along with possible pregnancy outcomes. She was immediately started on Metformin (1gm) and Biasp insulin (30) - 15 units at breakfast and 10 units at 
dinner. She was on Amlodipine for hypertension which was changed over to Nifedipine. She was advised to review after 1 week with a FBS, PPBS and HbA1c values. In the next visit her fasting was $187 \mathrm{mg} / \mathrm{dL}$, PP was $294 \mathrm{mg} / \mathrm{dL}$, $\mathrm{HbA} 1 \mathrm{c}-8.1 \%$, creatinine was 0.5 , and her $\mathrm{Bp}$ was $140 / 80$ $\mathrm{mm}$ of $\mathrm{Hg}$. Her dose of insulin was increased to 18 units before breakfast (BBF) and 12 units before dinner (BDN) and was again asked to review after 1 week. In the next visit, her FBS was $119 \mathrm{mg} / \mathrm{dL}$, after breakfast was 243 $\mathrm{mg} / \mathrm{dL}$, after lunch $120 \mathrm{mg} / \mathrm{dL}$, after dinner $150 \mathrm{mg} / \mathrm{dL}$. Her dose was insulin was then increased to 22 units at breakfast and 14 units at dinner and Metformin was continued. Insulin dosage was continuously adjusted according to the glycemic levels. The final prescription included Biasp insulin (30) 46 units at breakfast and 20 units at dinner and an insulin as part 7 units at lunch along with Metformin $1 \mathrm{gm}$ and Nifedipine was replaced with Labetalol 100mg. She delivered a single viable male child at 33 weeks of gestation due to pregnancy induced hypertension and type 2 diabetes mellitus with no abnormalities (Figure 1).

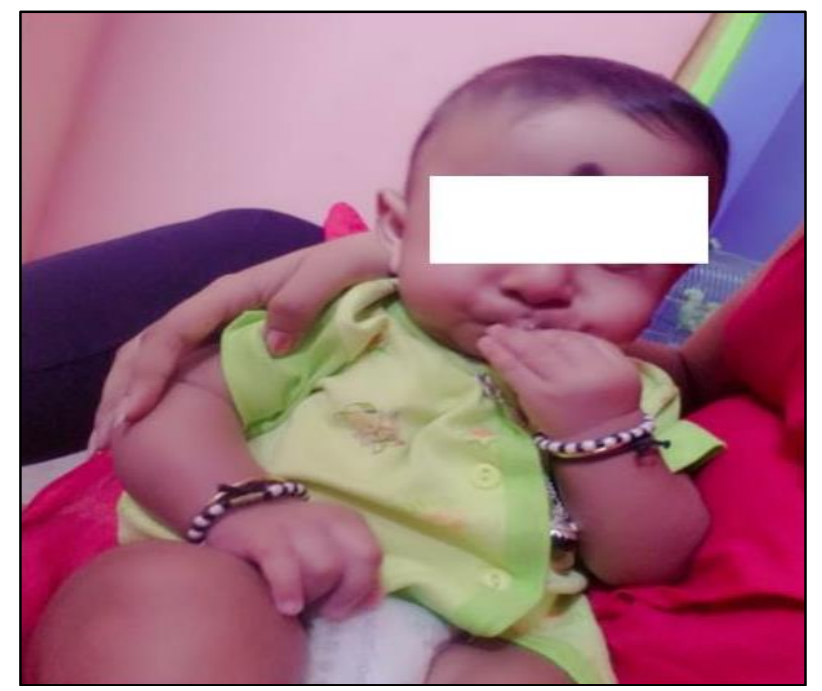

Fig 1: Healthy child with no congenial abnormality

\section{Discussion}

Insulin has traditionally been the first drug of choice for management of diabetes in pregnancy as because it does not cross the placenta and is highly efficacious in controlling blood sugar. Not a single study has been carried out in pregnant women so far with Pioglitazone so the risks or benefits associated with it's use cannot be assessed accurately. However, some animal studies were done which showed that it is associated with developmental delay, increased post implantation loss and so on. The same is the case with glimepiride wherein no clinical studies have been carried out assessing it's safety in pregnant women. As because of ethical and safety issues, it is not possible always to conduct a new study in pregnant women ${ }^{[5,6]}$. But recent data regarding metformin and glyburide suggests it's use in pregnant women or at the very least paves way for conducting more studies with it. As birth of a healthy child is very precious, adequate and fast control of hyperglycemia is required in pregnancy. Diet and lifestyles changes being the first step taken to control hyperglycemia in diabetes, it is not very much effective in pregnancy. So we are left with no other option except using insulin in such cases. Insulin offers a tighter control throughout pregnancy which in turn reduces the adverse outcomes associated with uncontrolled sugar levels. The patient when presented to us was already on glimepiride and pioglitazone and was already into 3rd month of pregnancy. We were in dilemma whether to continue with the pregnancy or not. We had informed the patient and her relatives of all the possible outcomes and closely monitored the patient's condition throughout the pregnancy till birth of the child. [7] One study done on pregnant rats using glimepiride showed improved blood glucose levels as well as favourable hematological and lipid profile. This calls for a more extensive clinical study to evaluate on the use of glimepiride in pregnant human females. We thoroughly reviewed the literature but couldn't find any reported use of pioglitazone and glimepiride in human pregnant cases.

\section{Conclusion}

In conclusion, we present the case of a type 2 diabetic patient who was treated with pioglitazone and glimepiride till 3rd month of pregnancy. The use of both of these agents is currently not approved during pregnancy. To our knowledge, this is the first case report on the use of these agents in pregnancy.

\section{Conflict of Interest \\ None}

\section{Acknowledgements}

None

\section{References}

1. American Diabetes Association: Management of diabetes in pregnancy Standards of medical care in diabetes-2018. Diabetes Care 2018;41:137-143.

2. Duckitt K, Harrington D. Risk factors for pre-eclampsia at antenatal booking: systematic review of controlled studies. BMJ 2005;330:565.

3. Bharti Kalra, Yashdeep Gupta, Rajiv Singla, Sanjay Kalra. N Am J Med Sci. 2015;7(1):6-12. doi: 10.4103/1947-2714.150081

4. Elliot BD, Langer O, Schenker S, Johnson RF. Insignificant transfer of glyburide occurs across the human placenta. Am J Obstet Gynecol 1991;165:807812 ,

5. Steve Hyer, Jyoti Balani, Hassan Shehata Int J Mol Sci. 2018;19(7):1954.Published online 2018 Jul 4. doi: 10.3390/ijms19071954

6. Thomas R. Moore: Glyburide for the Treatment of Gestational Diabetes, Diabetes Care, 2007;30(2) DOI: $10.2337 / \mathrm{dc} 07-\mathrm{s} 218$

7. Esubi JU, Olojede SO, Lawal SK. et al. Comparative Studies on Safety of Glimepiride and Glipizide on Renal Microarchitecture and Oxidative Stress Markers of Pregnant Streptozotocin-Induced Diabetic Wistar Rats. J Pharm Pharmacol Res 2019;3:003-018. 